\title{
Numerical and Experimental Studies on the Structure-Borne Noise Control on a Residential Kitchen Hood
}

\author{
Sinem Öztürk and Haluk Erol \\ Istanbul Technical University, Mechanical Engineering Faculty, Gümüşsuyu, 34439, Istanbul, Turkey.
}

\begin{abstract}
(Received 2 May 2011, revised 27 September 2012, accepted 28 November 2012)
\end{abstract}
The growing demand for highly efficient household appliances has driven the need for tools to predict, evaluate, and optimize both existing and new designs. Improving the design of the residential kitchen hood requires in-depth knowledge of the structure. In particular, the dynamic behaviour of the structure during the working period needs to be studied carefully during the design stage. A tool for predicting the structure-borne noise behaviour would save a considerable amount of time, reduce the number of prototypes that need to be built, and decrease the development costs. This paper concentrates on reducing the noise generated from the vibrating structure of a residential kitchen hood by using both numerical and experimental methods. Normal modes of the structure were identified, and the results agree well with the finite-element model. To validate the finite element model, an operational deflectionshape analysis of the structure was performed by using the laser Doppler vibrometry method. This study presents the finite element model and the experimental results of a kitchen hood. This study shows that the contribution of structure-borne noise from the vibrating panels to the overall kitchen hood noise levels is significant, especially at low frequencies. Thus, panel vibration is a critical design consideration for end users because of its relationship to noise and comfort.

\section{INTRODUCTION}

Residential kitchen ventilation is generally used on an intermittent basis. High levels of various contaminants accrue during cooking over a relatively short period of time. The contaminants must be removed quickly and completely, if possible. Kitchen range hoods have been introduced as a solution to these problems. The main function of a typical residential kitchen hood is ventilation. There is an increasing demand for highly efficient, reliable, stylish, and inexpensive range hoods due to the availability of new cooking appliances, and because occupants are increasingly sensitive regarding the issue of indoor air quality. In recent years, the kitchen hood market has become more competitive and requires quick release times for new designs. New designs are expected to be cheaper and to meet higher quality standards with respect to comfort and noise levels. Thus, engineers are required to find alternative design methods to satisfy the market. Once serial manufacturing has started, it is very expensive and difficult to change the design. Design changes can reach the customer within a time period of four months to two years, depending on the magnitude of the changes. Therefore, new methods are required so that the new designs can quickly respond to market conditions. These methods should reduce the engineering costs and must be reliable.

Computer-aided engineering has been widely used as a costeffective solution for testing new designs. However, the results from the computer-aided engineering software should be validated by using experimental studies. Failing to validate the computational methods can result in serious design errors. For this reason, computer-aided engineering studies should be conducted in parallel with experimental studies. Furthermore, in recent years, the capture efficiency of range hoods has increased. The capacity of the kitchen hood can be improved by increasing the drive unit, preferably without changing the standard external body dimensions. The main source of noise in kitchen hoods is the vibration that is generated by the drive unit, which consists of a variable speed electric motor and a fan, and by the air flow through the stationary duct. The vibration generated by the drive unit is transmitted to the house through connections. The connections have a complex vibroacoustical behaviour that is influenced by the various interaction mechanisms. An estimation of the noise produced from a vibrating structure is an important step in the design and development of high-quality kitchen hoods. It is very important to understand the dynamics of a kitchen hood before its construction begins. The most significant challenge that is encountered in noise and vibration control studies of residential kitchen hoods is that generalizing the results is impossible because of the large number of designs of residential kitchen hoods that are used in practice.

A literature review, however, reveals that most research on kitchen hood noise control has been performed by the manufacturers themselves. As a result, the number of publications on this topic is quite limited and may be biased. The first openly available study on the noise control of kitchen hoods was performed by Maggiorana et al. ${ }^{1}$ In the study, a combined experimental and theoretical methodology is proposed to address the problem of noise reduction of range hoods. The experimental approach is based on the use of simple microphones and accelerometers to generate a brief characterisation of the noise spectrum and the vibrational characteristics of different models of the product. The second phase of the study is based on theoretical modelling of the structural vibrations and flows by FEM and CFD codes to better understand and identify the primary sources of noise. Once the main problems were identified, some working prototypes of range hoods were created and the possibility of noise reduction was quantified by sound power estimation by using acoustical intensity measurement techniques.

In this study, the noise and the structural vibration behaviour of a residential kitchen hood was analysed by using numeri- 